\title{
Minihepcidins are rationally designed small peptides that mimic hepcidin activity in mice and may be useful for the treatment of iron overload
}

Gloria C. Preza, ${ }^{1}$ Piotr Ruchala, ${ }^{2}$ Rogelio Pinon, ${ }^{2}$ Emilio Ramos, ${ }^{3}$ Bo Qiao, ${ }^{2}$ Michael A. Peralta, ${ }^{4}$ Shantanu Sharma, ${ }^{5}$ Alan Waring, ${ }^{2,6}$ Tomas Ganz, ${ }^{1,2}$ and Elizabeta Nemeth²

${ }^{1}$ Department of Pathology, ${ }^{2}$ Department of Medicine, and ${ }^{3}$ Department of Chemistry and Biochemistry, UCLA, Los Angeles, California, USA. ${ }^{4}$ Department of Chemistry, Columbia University, New York, New York, USA. 5 Materials and Process Simulation Center, California Institute of Technology, Pasadena, California, USA. ${ }^{6}$ Department of Physiology and Biophysics, School of Medicine at the University of California, Irvine, Irvine, California, USA.

\begin{abstract}
Iron overload is the hallmark of hereditary hemochromatosis and a complication of iron-loading anemias such as $\beta$-thalassemia. Treatment can be burdensome and have significant side effects, and new therapeutic options are needed. Iron overload in hereditary hemochromatosis and $\beta$-thalassemia intermedia is caused by hepcidin deficiency. Although transgenic hepcidin replacement in mouse models of these diseases prevents iron overload or decreases its potential toxicity, natural hepcidin is prohibitively expensive for human application and has unfavorable pharmacologic properties. Here, we report the rational design of hepcidin agonists based on the mutagenesis of hepcidin and the hepcidin-binding region of ferroportin and computer modeling of their docking. We identified specific hydrophobic/aromatic residues required for hepcidin-ferroportin binding and obtained evidence in vitro that a thiol-disulfide interaction between ferroportin C326 and the hepcidin disulfide cage may stabilize binding. Guided by this model, we showed that 7-9 N-terminal amino acids of hepcidin, including a single thiol cysteine, comprised the minimal structure that retained hepcidin activity, as shown by the induction of ferroportin degradation in reporter cells. Further modifications to increase resistance to proteolysis and oral bioavailability yielded minihepcidins that, after parenteral or oral administration to mice, lowered serum iron levels comparably to those after parenteral native hepcidin. Moreover, liver iron concentrations were lower in mice chronically treated with minihepcidins than those in mice treated with solvent alone. Minihepcidins may be useful for the treatment of iron overload disorders.
\end{abstract}

\section{Introduction}

Hepcidin is a peptide hormone that mediates systemic iron homeostasis in vertebrates (1). Hepcidin controls plasma iron concentrations by inhibiting dietary iron absorption and release of recycled iron from macrophages. The hormone acts by inducing the endocytosis of its receptor ferroportin, the sole known cellular exporter of iron. Hepcidin deficiency causes or contributes to iron overload in several diseases, including hereditary hemochromatosis $(2,3), \beta$-thalassemia $(4,5)$, and chronic hepatitis $C(6,7)$.

Current treatment modalities for iron overload include phlebotomy and iron chelation. Phlebotomy is a relatively inexpensive and effective treatment for hereditary hemochromatosis but is not satisfactory for all patients. Some patients do not tolerate phlebotomy because of concurrent anemia, poor vascular access, adverse physiological responses to phlebotomy, fears, religious beliefs, and the lack of long-term convenient access to phlebotomy centers (8). Even the patients who are compliant with phlebotomy treatment may prefer oral therapy if it were available.

Conflict of interest: Tomas Ganz and Elizabeta Nemeth are officers and shareholders in Intrinsic LifeSciences, a company engaged in the development of iron-related diagnostic products.

Citation for this article: J Clin Invest. 2011;121(12):4880-4888. doi:10.1172/JCI57693.
In $\beta$-thalassemia, phlebotomy is generally not feasible because it worsens anemia, and iron overload is treated by regular chelation (9). Iron chelators have side effects ranging from mild to very serious, and compliance is often suboptimal. Iron overload remains a major cause of morbidity and mortality in $\beta$-thalassemia.

Additional therapeutic options are clearly desirable. Given the causal role of hepcidin deficiency in the development of iron overload, replacement of hepcidin would be a rational approach to the prevention and treatment of iron overload in these disorders (10). However, the use of natural hepcidin as a potential replacement therapy in hepcidin-deficient conditions has major limitations. Bioactive hepcidin is 25-amino acids long and has 4 disulfide bonds, rendering the production of a correctly folded full-length hepcidin expensive. The half-life of natural hepcidin is very short (11) due to its rapid renal excretion. Furthermore, peroral absorption of hepcidin would be low due to its large size $(\sim 2.7 \mathrm{kDa})$. In search of alternatives to full-length hepcidin, we carried out a structure-function analysis of the hepcidin-ferroportin interface. Based on this information, we developed a series of 7- to 9-amino acid peptides, "minihepcidins," that mimic the activity of hepcidin in cell-based bioassays and in mice. Our findings establish the feasibility of small, drug-like hepcidin agonists for the treatment of iron overload disorders due to hepcidin deficiency. 


\section{Results}

\section{Definition of the extracellular loop of ferroportin involved in bepcidin binding}

We previously showed that an extracellular ferroportin residue, C326, is essential for hepcidin binding (12), but the extent of the extracellular loop surrounding C326 was not known. In the 2 published models of ferroportin topology, the extracellular loop containing C326 spans the region from D325 to S342 (13) or F316 to T334 (14). We subjected the proposed ends of the extracellular loop to cysteine-scanning mutagenesis. In WT ferroportin, C326 and possibly C205 have extracellular location (13). Because of that, a double mutant (C205S/C326S) of human ferroportin-GFP was used as a template for site-directed mutagenesis to introduce cysteines in multiple positions between M312 and L345. To determine which of the newly introduced cysteines were located on the cell surface, HEK293T cells were transfected with plasmids encoding control or mutant ferroportin-GFP and biotinylated using nonpermeable, sulfhydryl (SH)-reactive biotin-maleimide. Streptavidin-peroxidase probing of SDS-PAGE blots of immunoprecipitated ferroportinGFP showed that, as expected, WT ferroportin-GFP but not the C205S/C326S double mutant was biotinylated (Figure 1A). Ferroportin mutants with cysteine substitutions in the region between M312 and L322 and between I344-L345 were expressed on the plasma membrane (Figure 1B) but were not biotinylated, suggesting that these residues are located in a transmembrane (or intracellular) region. In contrast, G323, F324, T334C, S338C, S340C, and S343C mutant ferroportins were all biotinylated, demonstrating their extracellular location and indicating that the extracellular loop containing C326 likely extends from G323 to S343.

A previous study (15) described the F324-S343 ferroportin region as the hepcidin-binding domain (HBD) and reported that a synthetic peptide corresponding to the HBD interacts with hepcidin in a manner similar to that of the membrane-associated cellular ferroportin. However, in our studies, the synthetic HBD peptide (RR-FDCITTGYAYTQGLSGSILS-RR) did not bind hepcidin specifically. As analyzed by surface plasmon resonance, synthetic human hepcidin interacted equally with the WT and C326S HBD peptide (Supplemental Figure 1A; supplemental material available online with this article; doi:10.1172/JCI57693DS1) or with unalkylated and alkylated HBD (data not shown). In contrast, similar modifications to ferroportin have dramatic effects on hepcidin binding (12). Any interactions that were observed between human hepcidin and HBD were nonspecific and probably due to hepcidin aggregating readily on a variety of surfaces (Supplemental Figure 1B). The reverse configuration, in which hepcidin was coated on the chip and HBD was in fluid phase, did not result in any binding (Supplemental Figure 1C). The results indicate that the synthetic F324-S343 peptide does not bind hepcidin specifically and that the segment does not function as an autonomous HBD.

\section{Ferroportin residues in the vicinity of $\mathrm{C} 326$ also participate in bepcidin binding}

To determine whether ferroportin residues other than C326 contribute to hepcidin binding, we mutagenized to alanine each residue in the C326 extracellular loop of WT human ferroportin (Fpn)GFP except for A332, which was substituted with D (aspartate). HEK293T cells were transfected with the ferroportin mutants, and the uptake of radiolabeled hepcidin was measured. To account for the variability of transfection efficiency, radioactive counts were normalized to the expression of ferroportin-GFP as determined by Western blotting. D325A protein was expressed at very low levels, and we therefore tested the mutant D325N, which had a normal level of expression. Remarkably, substitutions in the first half of the extracellular loop markedly reduced hepcidin binding, whereas mutations in the second half did not interfere with hepcidin binding (Figure 1C). However, a reduction in hepcidin binding by a specific mutation may occur not only because the residue is involved in contacting hepcidin but because of mistrafficking of the mutant protein or because of a conformational change, leading to reduced accessibility of the binding site containing C326. To address these possibilities, we used $\mathrm{SH}$-reactive biotin-maleimide to biotinylate cells transfected with the ferroportin-GFP mutants from Figure 1C. The mutations that reduced hepcidin binding without affecting the accessibility of C326 were F324A and Y333A but not D325N, I327A, and A332D (Figure 1D). Thus, F324 and Y333 likely make direct contact with hepcidin. The fluorescence microscopy images of the mutants' cellular localization are shown in Supplemental Figure 2.

\section{Identification of hepcidin residues critical for binding to ferroportin} We showed previously that deletion of the $5 \mathrm{~N}$-terminal residues of hepcidin ablated the ability of the peptide to cause ferroportin degradation (16). By substituting individual $\mathrm{N}$-terminal residues (residues 1-6 and 8), we also showed that $\mathrm{H} 3, \mathrm{~F} 4$, and $\mathrm{I} 6$ were important for the peptide activity and that those positions required hydrophobic side chains for interaction with ferroportin (17). Here, we mutagenized the next large hydrophobic residue, F9. Substitutions of F9 with alanine or cyclohexylalanine (a nonaromatic hydrophobic residue) resulted in 100- and 10-fold reduced activity respectively, suggesting that position 9 requires an aromatic side chain (Figure $2 \mathrm{~A}$ ).

Because our previous study on the ferroportin thiol residue C326$\mathrm{SH}$ (12) indicated that disulfide bond formation may occur during hepcidin-ferroportin interaction, we next focused on the effect of individual disulfide bond substitutions on hepcidin peptide activity. Although we previously partially explored this question (16), the hepcidin structure has since been revised (18), and we generated synthetic hepcidin mutants in which we substituted pairs of cysteines with alanines according to the revised connectivity (C7A/C23A, C10A/C13A, C11A/C19A, C14A/C22A). A dose-response analysis of the mutant peptide activity (Figure $2 \mathrm{~B}$ ) showed that all these mutants had a similar decrease in activity (up to 100 fold). Circular dichroism spectroscopy indicated that the mutant peptides had slightly more disordered structure than the native peptide (Supplemental Figure 3), perhaps partially accounting for the diminished activity. Given that none of the mutants displayed a complete loss of activity, the result suggests that if disulfide exchange is involved in hepcidin binding, more than one disulfide bond could participate in exchange with C326-SH on ferroportin.

\section{RosettaDock computer modeling}

To gain further insight into the hepcidin-ferroportin interaction, we performed computer modeling. The input structures were the fulllength hepcidin (PDB 2KEF), with the most recent disulfide bond assignment (18), and a region of ferroportin (residues 306-362) encompassing the C326 extracellular loop and flanking transmembrane helices, with structure based on a recently developed theoretical model of ferroportin molecule (19). Ferroportin and hepcidin structures were refined using HyperChem 7.5 and GROMACS 


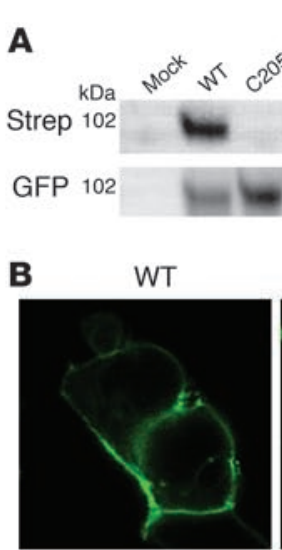

Y318C
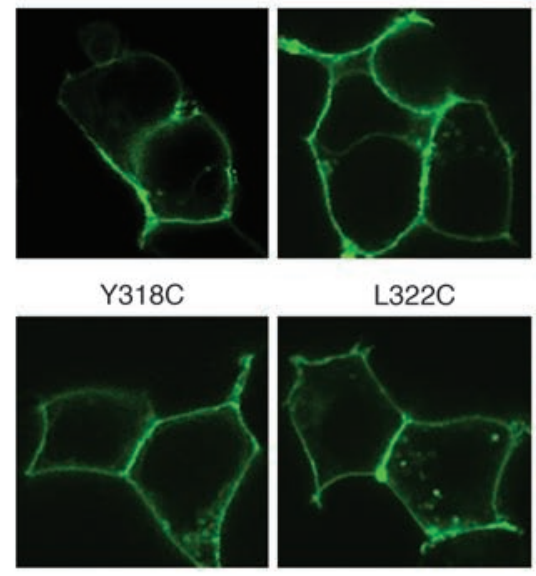

L322C
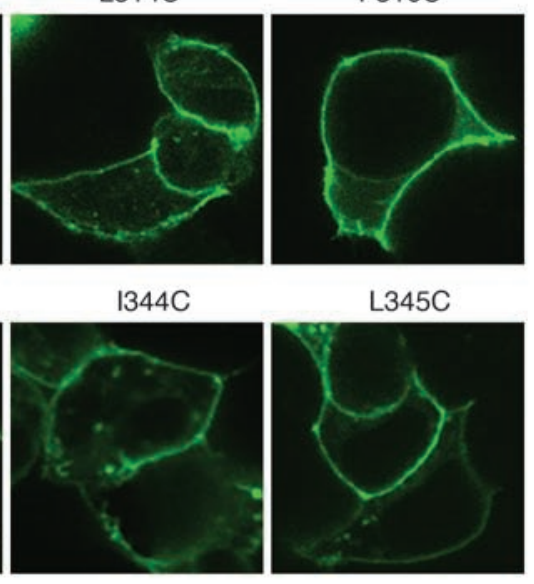

L345C

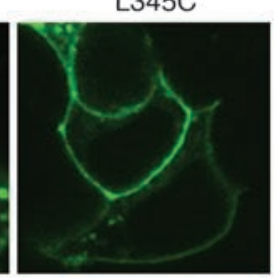

C

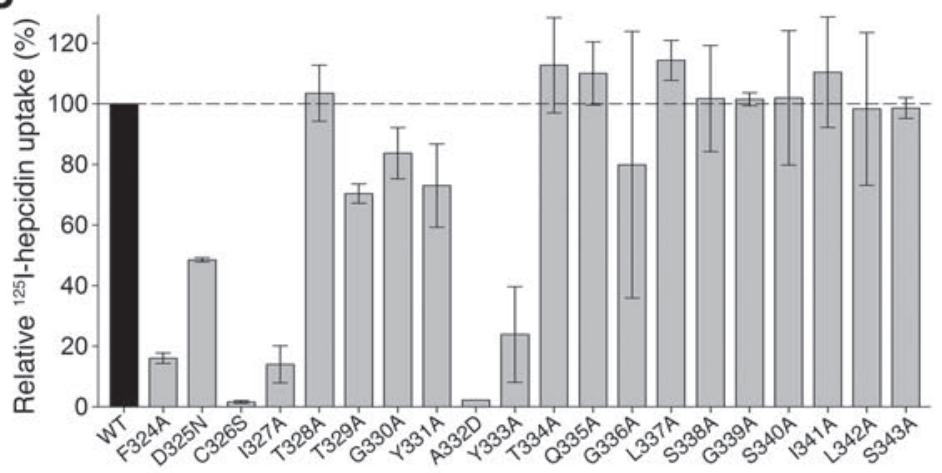

D

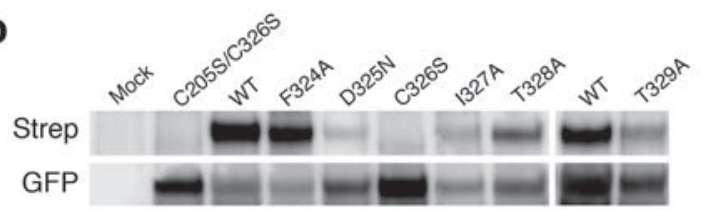

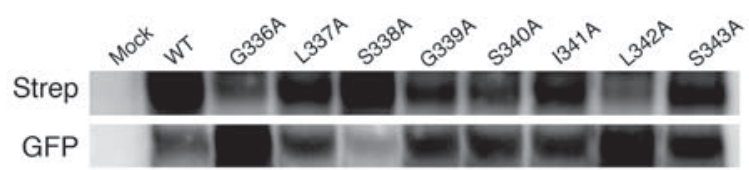

\section{Figure 1}

Defining the hepcidin-binding loop on ferroportin. (A) To detect the boundaries of the C326-containing extracellular loop, certain residues between M312 and L345 on the human Fpn-GFP C205S/C326S mutant were substituted with cysteine. Mutant constructs were transfected into HEK293T cells, and cell-surface biotinylation was performed using a nonpermeable, SH-reactive biotin-maleimide. Protein lysates were immunoprecipitated with anti-GFP antibody, analyzed by SDS-PAGE, blotted, and probed with streptavidin-peroxidase to determine which mutated residues had extracellular localization. The bottom row indicates the amount of FpnGFP that was immunoprecipitated. Each mutant was tested in at least 3 separate experiments. (B) Confocal microscopy of HEK293 cells transfected with cysteine substitution mutants. Mutant proteins M312C, L314C, F316C, Y318C, L322C, I344, and L345 were all displayed on the cell surface similar to WT ferroportin-GFP, indicating that mistrafficking was not the reason for the absence of cell-surface biotinylation in A. Original magnification, $\times 63$. (C) To define the contribution of residues in the C326 extracellular loop to hepcidin binding, alanine-scanning mutagenesis and other substitutions were performed as indicated. After transfection of HEK293T cells, cellular uptake of ${ }^{125}$ I-hepcidin was determined and normalized to the Fpn-GFP levels in each sample, determined by Western blotting. Each bar represents the average of 3 to 4 separate experiments, and error bars indicate standard deviation. (D) Cell-surface biotinylation of constructs from C. Of the mutants that showed decreased hepcidin uptake, only F324A and Y333A mutants had thiol-specific biotinylation similar to that of WT Fpn-GFP, whereas others (D325N, I327A, A332D) had decreased cell-surface thiol-biotinylation, suggesting that these mutants may be mistrafficked or that a conformational change may be blocking the C326-SH accessibility. before docking studies. The initial stage docking program ZDOCK (20) was first used to identify the lowest energy conformer set, and this ensemble was optimized using the RosettaDock program (21). The RosettaDock refinement run generated 1,000 structures whose energy scores were plotted versus the root mean square distance from the starting input conformation (Figure 3A). The plot showed the optimal energetic "funnel" of low-energy structures. The 10 best-scoring low-energy structures all had the $\mathrm{N}$ terminus of hepcidin as the part forming the interface with ferroportin. Of those 10 structures, the conformation most compat- 
A

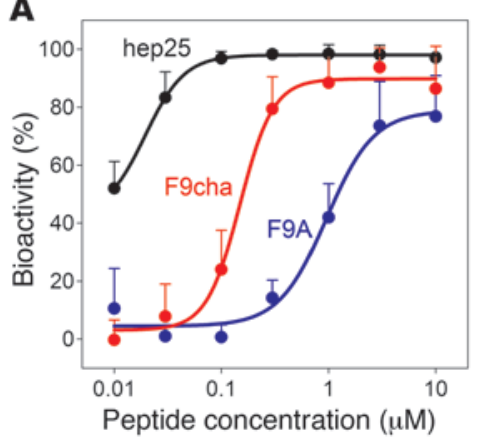

B

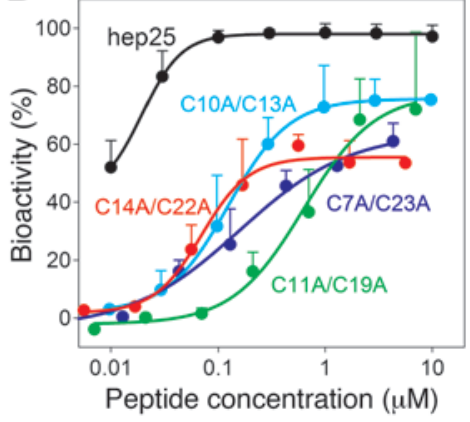

\section{Figure 2}

Hepcidin amino acid residues important for binding to ferroportin. Full-length 25-amino acid hepcidin variants were prepared by chemical synthesis and oxidatively refolded. The peptide activity was measured by a flow cytometry-based assay detecting ferroportin-GFP degradation. (A) F9 substitution with nonaromatic cyclohexylalanine (cha) or alanine caused a 10- and 100-fold decrease in peptide activity. (B) Pairwise substitutions of cysteines with alanines to remove individual disulfide bonds reduced bioactivity to a similar extent. Each data point is the average of 3 to 4 individual experiments and error bars indicate SD. ible with our mutagenesis data (second-lowest energy structure) was used as the final hypothetical model of the ferroportin-hepcidin complex (Figure 3B and Supplemental Figure 4).

The model highlighted that aromatic and hydrophobic side chain interactions can drive the hepcidin-ferroportin interaction (Supplemental Table 1). In the model, H3, F4, and I6 of hepcidin formed a hydrophobic pocket for the interaction with Y333 of ferroportin, and F9 of hepcidin interacted with F324 of ferroportin through pi-stacking, in agreement with our mutagenesis data. The model also positioned the $\mathrm{C} 326$ residue in close proximity to the hepcidin disulfide framework.

\section{Minibepcidins display agonist activity}

The mutagenesis approach as well as RosettaDock modeling suggested that hepcidin residues important for binding to ferroportin are located at the highly conserved $\mathrm{N}$ terminus of the peptide and prominently include residues $\mathrm{H} 3, \mathrm{~F} 4, \mathrm{I} 6$, and F9. We thus synthesized short peptides (minihepcidins), consisting of up to $9 \mathrm{~N}$-terminal amino acids of hepcidin (Supplemental Table 2), and tested their ability to cause ferroportin-GFP degradation in the cellular bioassay. These first generation minihepcidins showed substantial agonist activity (Figure 4), although they were less potent than the full-length hepcidin $\left(\mathrm{EC}_{50}=9 \mathrm{nM}\right)$. The minihepcidin containing $9 \mathrm{~N}$-terminal residues (hep9, DTHFPICIF; $\mathrm{EC}_{50}=76 \mathrm{nM}$; Supplemental Figure 5) was more active than shorter minihepcidins.

\section{Figure 3}

A RosettaDock model of the interaction between hepcidin and the ferroportin loop surrounding C326. (A) Plot of the full set of 1,000 structures generated in the RosettaDock refinement run. The energy score versus the root mean square (rms) distance from the starting structure shows the optimal energetic funnel of low-energy structures clustered around a single position. The plot indicates that the docking algorithm has reached a good local minimum and that the lowest scoring (lowest free energy) structures are the most stable. The second-lowest energy structure was most compatible with our mutagenesis data and is presented in B. (B) Hepcidin sequence is shown in pink, with yellow disulfide connectivities. Ferroportin extracellular loop is shown in cyan, transmembrane helices are shown in gray, and the sulfur of C326 is shown in yellow. The strongly interacting side chain pairs are displayed in thicker lines. Hydrogens are not shown. The model identified hydrophobic interactions between $\mathrm{H} 3, \mathrm{~F} 4$, and 16 on hepcidin and $\mathrm{Y} 333$ on ferroportin and pi-stacking interaction between F9 and F324. The interaction of aromatic residues on the hepcidin and ferroportin surfaces brings C326 close to the disulfide network of hepcidin (disulfide cage), raising the possibility of transient disulfide exchange.

\section{Thiol cysteine is required for minihepcidin activity}

Because our previous study showed that a thiol form of the C326 residue on ferroportin was necessary for hepcidin binding and that the mutant with isosteric substitution C326S did not bind hepcidin in vitro (12) and caused hepcidin resistance in vivo (22), we hypothesized that a disulfide exchange between C326 and the disulfide framework of hepcidin may occur upon hepcidin binding to ferroportin. To determine whether disulfide bonding could also be involved in minihepcidin binding to ferroportin, we tested the requirement for cysteine on minihepcidin by mutating the $\mathrm{C} 7$ residue of hep9. We found that the activity of hep9 depended on its ability to participate in disulfide exchange. Isosteric substitution with serine (C7S) or blocking of C7 thiol with a t-butyl group (C7-S-t-but), which prevents disulfide exchange, ablated the agonist activity of hep9 (Figure 5A). However, the activity was considerably preserved when the blocking group was disulfide linked to C7 (C7-SS-t-but) and was therefore capable of disulfide exchange.

These results strongly suggest that the minihepcidin-ferroportin interaction is dependent on the thiol-thiol interaction or
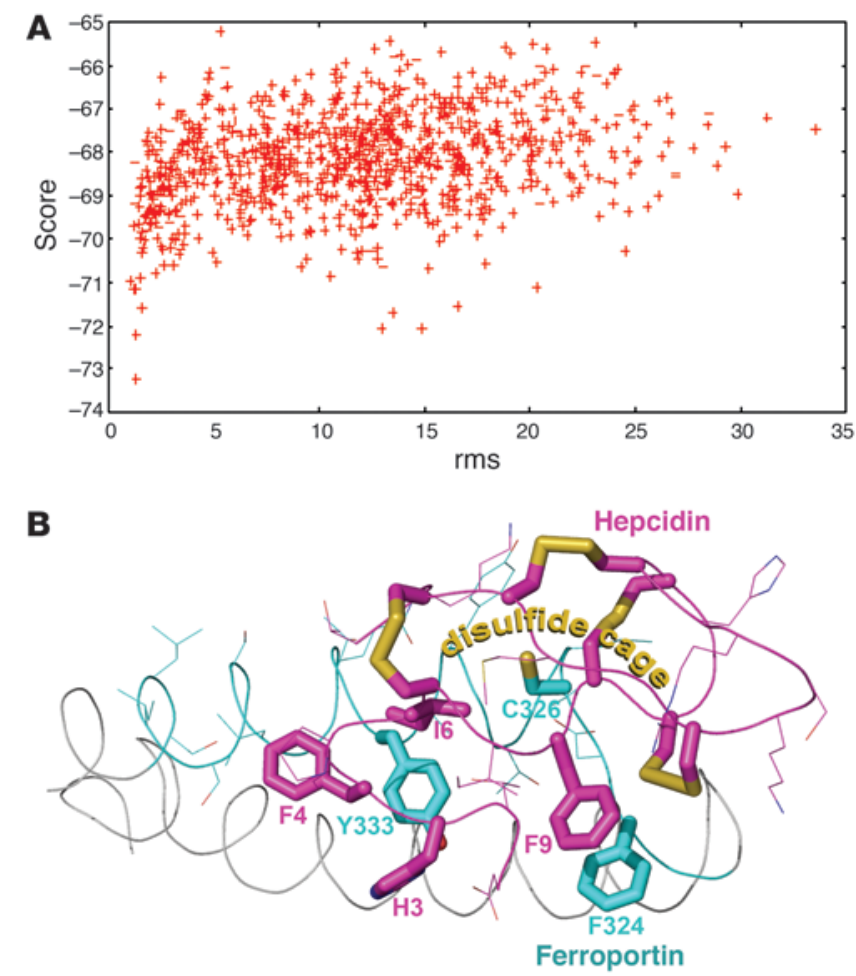

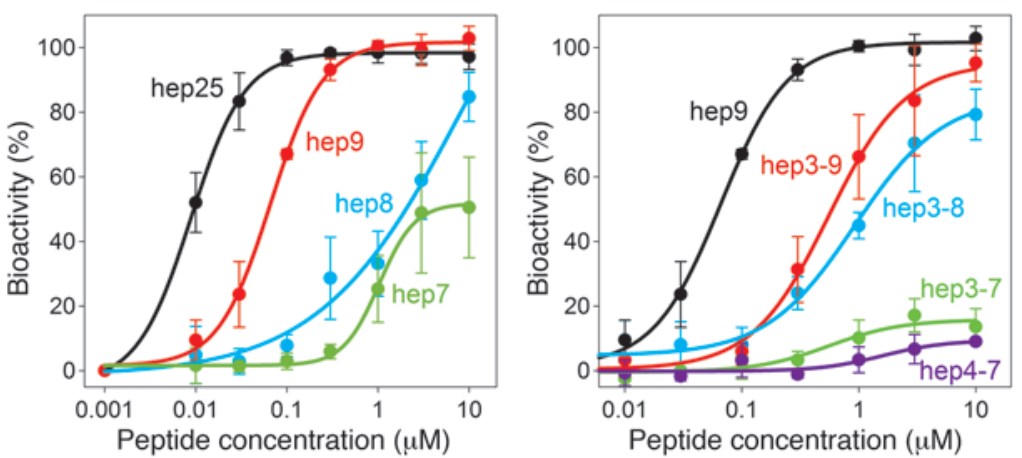

Figure 4

Minihepcidins are effective hepcidin agonists. The activity of synthetic minihepcidins was determined by flow cytometric quantitation of ferroportin-GFP degradation in stably transfected HEK293 cells. Each point is an average of 5 to 6 experiments and error bars indicate SD. hep8, DTHFPICl; hep7, DTHFPIC; hep3-9, HFPICIF; hep3-8, HFPICl; Hep3-7, HFPIC; Hep4-7, FPIC. disulfide formation and that the hepcidin-ferroportin interaction could involve disulfide exchange. To further examine this, we tested whether the hepcidin-ferroportin complex is affected by reducing agents. In our previous experiments, we noted that ${ }^{125}$ I-hepcidin forms a complex with ferroportin that does not dissociate during protein extraction, immunoprecipitation, and SDS-PAGE. However, this complex readily dissociated in the presence of DTT (Figure 5B), supporting the possibility of hepcidinferroportin disulfide exchange.

\section{Design and in vitro bioassay of minihepcidins}

In order to optimize the agonist activity, we designed additional minihepcidins of varying lengths and structural modifications (Supplemental Table 2). All peptides were synthesized as carboxyamides $\left(-\mathrm{CONH}_{2}\right)$ to make their $\mathrm{C}$ terminus similar to the corresponding intact hepcidin segment. The peptide activity was tested in a cellular bioassay in vitro, measuring their ability to cause degradation of ferroportin-GFP (16).

Substitution or stabilization of the C7 thiol group in hep 9 decreases bioactivity. To increase the stability of minihepcidins, we synthesized circular peptides by introducing an internal disulfide bond with C7. We also modified the size and reactivity of the amino acid corresponding to C7 by introducing various cysteine analogs at that position. All of these cysteine analogs showed decreased bioactivity relative to that of hep9.

Retro-inverso minihepcidins are bioactive. To increase the resistance of minihepcidins to proteolysis in vivo, we introduced unnatural amino acids (chemical structures shown in Supplemental Table 3) or synthesized retroinverso analogs. Retro-inverso peptides are composed of $\mathrm{D}$-amino acids assembled in the reverse order from that of the parent L-peptide sequence, making them sterically similar to the original but much more resistant to proteolysis. Retro-inverso hep9 analog (ri-hep9, FICIPFHTD) and retro-inverso hep3-9 (ri-hep3-9, FICIPFH) caused ferroportin degradation in vitro, although with several fold lower activity than that of hep9.

Effects of other peptide modifications. Additional peptide variants were pegylated in order to increase solubility, reduce immunogenicity, and increase circulatory time by reducing renal clearance. To increase the intestinal uptake of minihepcidins via enterohepatic circulation of bile acids, the pegylated retro-inverso hep 9 was conjugated with chenodeoxycholic or ursodeoxycholic bile acids (cheno-ri-hep9 and urso-ri-hep9). Cheno-ri-hep9 had similar activity in vitro to that of unmodified hep9, while ursori-hep9 had 10-fold lower activity. We also synthesized retro-inverso hep9 conjugated to palmitoyl groups because palmitoylation of peptides could increase intestinal absorption, reduce renal excretion through increased binding to albumin, and potentially increase affinity for cell membranes (23). Singly palmitoylated retro-inverso hep9 (palmitoyl-ri-hep9) was as active as hep9, but the peptide with 2 palmitoyl groups had no activity. Beta-propeller and collagen domain modifications, designed to multimerize minihepcidins and thereby reduce their renal excretion, resulted in inactive minihepcidins.

\section{Minibepcidins induce hypoferremia in mice}

Several of the peptides that were most active in vitro were selected for testing in mice. Although our minihepcidins were derived from the human sequence, the human hepcidin is active in the mouse presumably because of the high degree of identity between mouse and human sequences of hepcidin and ferroportin. Selected ana-

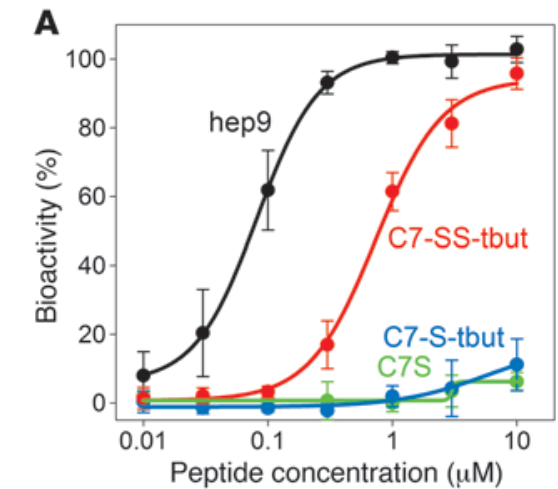

Figure 5

Evidence for the role of disulfide exchange in the hepcidin-ferroportin interaction. (A) The agonist activity of minihepcidin depends on its thiol reactivity. Hep9 was modified at residue $\mathrm{C}$; ; $\mathrm{C} 7$-SS-tbut contains an exchangeable tert-butyl group disulfide-linked to C7; C7-S-tbut contains a tert-butyl group in a thioether linkage to $\mathrm{C} 7$, which prevents disulfide exchange; and C7S contains a serine in position 7 instead of a cysteine. Each data point is the average of 3 to 4 individual experiments and error bars indicate SD. (B) Reducing agents disrupt ${ }^{125}{ }^{-h e p c i d i n}$ association with Fpn-GFP. ${ }^{125}$ I-hepcidin (125I-hep) was added to cells expressing ferroportin-GFP for 15 minutes, and total cellular protein was immunoprecipitated using anti-GFP antibody. The sample was split into 2 identical aliquots, and DTT was added to 1 of the aliquots (final concentration $100 \mathrm{mM}$ ). The amount of immunoprecipitated Fpn-GFP was determined by Western blotting. Similar experiments were performed at least 5 times. 

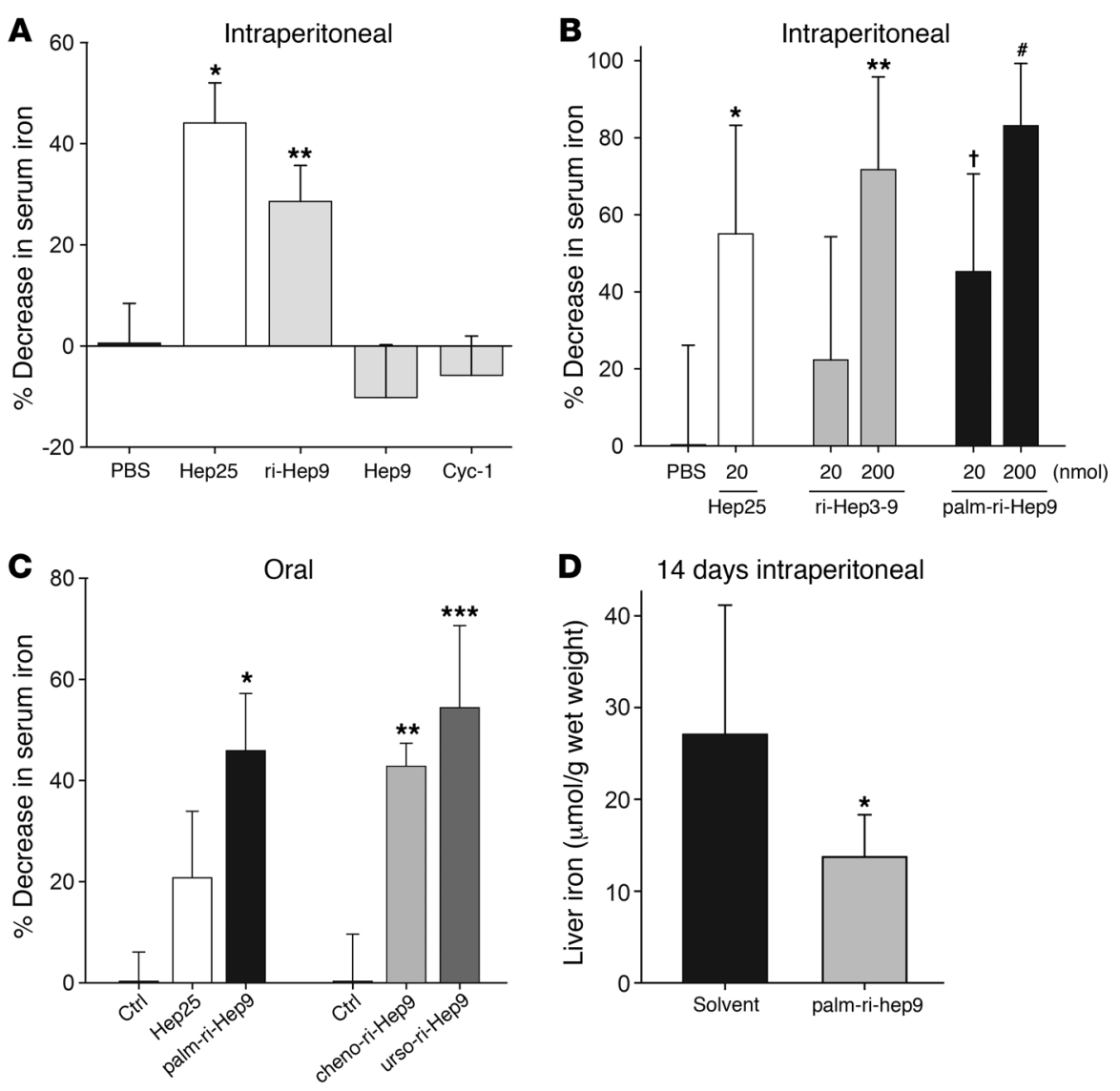

D

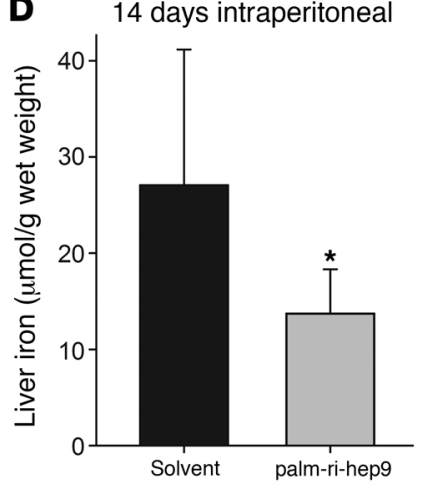

\section{Figure 6}

Bioactivity of parenteral and oral minihepcidins in mice. (A) Full-length hepcidin (20 nmoles), minihepcidins (200 nmoles), or diluent were injected intraperitoneally ( $n=6$ animals per treatment), mice were euthanized after 4 hours, and nonheme iron levels were determined in serum. ri-hep9, retro-inverso hep9; Cyc-1, minihepcidin with a disulfide bond (CDTHFPICIF). ${ }^{*} P=0.003$ and ${ }^{* \star} P=0.035$ in comparison with control mice. (B) Full-length hepcidin (20 nmoles), minihepcidins (20 and 200 nmoles), or diluent were injected intraperitoneally into mice ( $n=5-7$ per treatment), and their serum iron was measured after 4 hours. ri-hep3-9, retro-inverso HFPICIF. ${ }^{\star} P=0.02 ;{ }^{* \star} P=0.002$; $\dagger P=0.015 ; \# P<0.001$. (C) Peptides $(200$ nmoles) or diluent were administered orally by gavage, and serum iron measured was after 4 hours. The results are expressed as the percentage of decrease in serum iron as compared with the serum iron levels in PBS-treated mice to normalize for the variations in absolute serum iron levels between experiments. ${ }^{*} P=0.006$; ${ }^{* *} P=0.003$; ${ }^{* \star *} P=0.01$. (D) Hepcidin-1 knockout mice were injected intraperitoneally with palmri-hep9 (100 nmoles per mouse; $n=7$ ) or diluent $(n=6)$ daily for 2 weeks. Liver iron content was measured on day $15 .{ }^{*} P=0.03$. Error bars represent SD. logs were injected intraperitoneally into mice, and a decrease in serum iron was measured 4 hours after injection. Control mice were treated with the diluent or with 20 nmoles human hep25 ( $50 \mu \mathrm{g}$ per mouse). Even at 10-times higher concentrations, minihepcidin hep 9 had no effect on serum iron levels, presumably because it was rapidly degraded, but a more stable retro-inverso hep9 showed significant activity $(P=0.028$; Figure 6A). Minihepcidins conjugated to fatty or bile acids were even more active. Injection of 20 nmoles palmitoyl-ri-hep 9 caused almost as large a decrease in serum iron levels as the equivalent dose of hep 25 (Figure 6B). Importantly, when the cysteine residue in palmitoylri-hep9 was substituted with serine, no serum iron decrease was observed (Supplemental Figure 6). Injection of a higher dose of palmitoyl-ri-hep9 (200 nmoles) caused more profound hypoferremia than did injection of hep 25 , with an $80 \%$ drop in serum iron (Figure 6B). Even a 7-amino acid retro-inverso peptide (ri-hep3-9; Figure $6 \mathrm{~B}$ ) was bioactive after parenteral injection, causing a $70 \%$ decrease in serum iron at 200 nmoles. The decrease in serum iron was not a consequence of the induction of endogenous hepcidin, as murine hepatic hepcidin-1 mRNA levels measured by qRT-PCR were not increased in agonist-treated mice compared with those in controls (Supplemental Figure 7).

\section{Selected minihepcidins are bioactive when administered by gavage}

In many situations, the most desirable method of drug delivery is by the peroral route. To test whether minihepcidins administered orally would exert a hypoferremic effect, we gavaged mice with 200 nmoles retro-inverso hep 9 that was palmitoylated or conjugated to bile acids (chenodeoxycholic or ursodeoxycholic acid). All 3 minihepcidins caused a significant decrease in serum iron compared with that in controls gavaged with diluent or with Hep25 (Figure 6C).

\section{Minihepcidins prevent liver iron overload in hepcidin-1 knockout mice}

In a proof-of-principle experiment, we examined the chronic effect of minihepcidin injections on liver iron accumulation in a mouse model of hereditary hemochromatosis. We chose hepcidin-1 knockout mice to eliminate any possibility of endogenous hepcidin contributing to the regulation of iron loading. Hepc1-/animals were injected parenterally with palmitoyl-ri-hep9 (100 nmoles; $n=7)$ or diluent $(n=6)$ daily for 2 weeks. The end point hemoglobin $(15 \pm 2 \mathrm{~g} / \mathrm{dl}$ and $15.9 \pm 1.5 \mathrm{~g} / \mathrm{dl}$ in palmitoyl-ri-hep9 and control group, respectively) and other hematological parameters were similar between the 2 groups of mice, indicating that minihepcidin injections did not cause iron-restricted erythropoiesis. However, the nonheme iron concentration of the liver was significantly lower in animals treated with the minihepcidin (13.7 \pm 4 $\mu$ moles/g wet weight) than that in those treated with diluent only (27.1 $\pm 13.7 \mu$ moles $/ g$ wet weight $)(P=0.03$; Figure 6D).

\section{Discussion}

In hereditary hemochromatosis, hepcidin deficiency is a consequence of destructive mutations in the genes encoding hepcidin (24) or one of its regulators, HFE (3) (most common), transferrin receptor 2 (25), or hemojuvelin (26). Hepcidin deficiency results 
in excessive intestinal iron absorption and maldistribution of tissue iron. If severe and untreated, the disease can progress to cirrhosis, hepatocellular carcinoma, and endocrine and cardiac problems. In the US, about $0.4 \%$ of people of mixed European descent have hereditary hemochromatosis mutations that put them at risk for iron overload (27).

Iron overload also occurs in $\beta$-thalassemia, a disease that affects millions of patients whose origins are in the Mediterranean basin and Asia, regions historically affected by endemic malaria (28). In $\beta$-thalassemia, hepcidin is suppressed by increased erythropoietic activity through mechanisms that are still incompletely defined. In the absence of transfusions ( $\beta$-thalassemia intermedia), hepcidin levels are very low $(4,5)$, and patients develop iron overload similar to that of severe hereditary hemochromatosis and may succumb to iron overload if untreated (29). In $\beta$-thalassemia major, transfusions rather than dietary iron absorption are the predominant cause of iron overload. Here, hepcidin levels are higher, because ineffective erythropoiesis is suppressed by transfusions and also because the additional iron load stimulates hepcidin production. However, in the intervals between transfusions, hepcidin concentrations progressively decrease toward the end of the interval $(4,5$, 30 ), and this recurrent lowering of hepcidin may also contribute to iron overload in $\beta$-thalassemia major.

Milder hepcidin insufficiency is seen in chronic liver disease, including viral hepatitis $(6,7,31)$. The cause of hepcidin suppression in these diseases is not yet clear, but the resulting chronic iron loading in the liver worsens the prognosis (32).

Therapeutic augmentation of hepcidin levels would be expected to curb hyperabsorption of dietary iron in these patients. The proof of this concept was achieved in mouse model of HFE hereditary hemochromatosis, in which the introduction of a hepcidin transgene into $\mathrm{Hfe}^{-/-}$mice prevented the development of iron overload (33). Hepcidin also causes redistribution of iron when iron overload is already established. Hepcidin induction in HFE-null mice carrying a Tet-inducible hepcidin construct did not acutely reverse the iron overload but did shift the excess iron from hepatocytes and other parenchymal cells to macrophages (34). Macrophages are relatively resistant to the toxic effects of iron (35), as demonstrated by the relatively benign course of "ferroportin disease," in which iron accumulates in macrophages. Most affected patients have no clinical manifestations, despite often severe iron overload (36). Thus, hepcidin-mediated redistribution of iron from parenchyma to macrophages in iron-overloaded patients could potentially limit iron toxicity in the heart, the pancreas, and the liver.

In addition to the beneficial effect of hepcidin on iron balance and distribution, recent studies suggest that hepcidin may also improve disordered erythropoiesis in $\beta$-thalassemia. Although the mechanism is still not understood, transgenic expression of hepcidin in a mouse model of $\beta$-thalassemia intermedia increased hemoglobin and decreased extramedullary erythropoiesis (37).

Using full-length hepcidin for the treatment of iron overload conditions would be expected to be expensive, not only because the synthesis and refolding of hepcidin with 4 disulfide bonds allows for a large number of alternative folds but also because of the relatively high dose required for its biological effect. Even assuming that hepcidin could be produced at a cost comparable to that of recombinant insulin, a typical dose of hepcidin would likely be many fold higher than that of insulin. In human patients with type 1 diabetes mellitus (and mouse models), a typical dose of insulin is $0.2-0.7 \mathrm{U} / \mathrm{kg} / \mathrm{d}$ or $9-32 \mu \mathrm{g} / \mathrm{kg} / \mathrm{d}$ (1 U is the biological equivalent of $45.5 \mu \mathrm{g}$ pure crystalline insulin). A typical dose of hepcidin, on the other hand, is $50 \mu \mathrm{g} / \mathrm{mouse} / \mathrm{d}$ or $2 \mathrm{mg} / \mathrm{kg} / \mathrm{d}$, which is nearly 100 -times higher than the typical dose of insulin. Given the current limitations of peptide synthetic technology, designing more potent and less expensive hepcidin analogs would be advantageous.

In this study, we developed small peptides, minihepcidins, that act as hepcidin agonists. Their rational design was facilitated by the identification of the region on hepcidin and ferroportin molecules that is critical for their binding. Together with our previous structure-function studies $(16,17)$, we showed that hydrophobic contacts dominate the ligand-receptor interaction and that several $\mathrm{N}$-terminal amino acids of hepcidin, up to residue 9 , are critical for its activity. We therefore used the first 9 residues as the scaffold for minihepcidin design. We also found that the ability to participate in thiol-thiol interactions is essential for minihepcidin activity. Blocking the only cysteine (C7) with a protective group abrogated the peptide activity unless the protective group was added in such a way that disulfide exchange was possible. Furthermore, in contrast to its Cys-containing counterpart, minihepcidin bearing the Cys to Ser substitution did not cause serum iron decrease in mice after intraperitoneal injection.

To improve agonist activity, we developed the minihepcidins with structural modifications that addressed some undesirable physicochemical properties of the minihepcidin scaffold, such as thiol instability, hydrophobicity, poor gastrointestinal absorption, susceptibility to proteolysis, and instability in the bloodstream. We succeeded in designing minihepcidins of 7- or 9-amino acids in length that were active in mice in vivo. Intraperitoneal injection of proteolysis-resistant retro-inverso minihepcidins caused hypoferremia similar to that caused by native hepcidin. In a proofof-principle experiment, chronic administration of minihepcidins significantly decreased iron loading in a mouse model of hereditary hemochromatosis. Hepcidin-1 knockout mice, which received intraperitoneal injections of a retro-inverso minihepcidin daily for 2 weeks, had significantly lower liver iron content that hepcidin-1 knockout mice injected with solvent.

Importantly, minihepcidin conjugated to fatty or bile acids caused hypoferremia after oral administration by gavage also. Given the number and rich variety of peptides involved in biological processes, relatively few have been FDA approved as therapeutics, and their efficient delivery by the oral route is still an unmet goal. Development of oral hepcidin analogs would represent a major advance in peptide pharmacology.

The mainstay of treatment for patients with iron overload is phlebotomy if they are not anemic and iron chelation if they have anemia. Although these measures are effective at reducing excess iron, they are frequently not well tolerated by patients, and compliance with iron-depleting therapy is suboptimal. If hepcidin therapy proves to be effective and relatively free of side effects, it could represent a major improvement over existing therapies, either alone or in combination with current approaches to allow modifications that would make the treatment less burdensome and better accepted by patients.

\section{Methods}

Site-directed mutagenesis. For cysteine scanning of the ferroportin region M312-L345, the human ferroportin-GFP C205S/C326S mutant (12) was used as a template for site-directed mutagenesis. For alanine scanning of the region F324-S343, WT human ferroportin-GFP was used as a template. Primers were selected using the QuikChange Primer Design (https://www. 
genomics.agilent.com/), and mutagenesis was performed using QuikChange II XL Mutagenesis Kit (Agilent Technologies). Mutant plasmids were sequenced to verify the presence of the mutations.

Transient transfection and microscopy. WT and mutant ferroportin-GFP plasmids were transiently transfected into HEK293T cells using either Lipofectamine 2000 (Invitrogen) or Amaxa nucleofection (Lonza) (12). Cells were visualized with an epifluorescence microscope (Nikon Eclipse), and images were acquired with a SPOT camera and SPOT Advanced Imaging Software (Diagnostic Instruments). Confocal microscopy was performed at the UCLA CNSI Advanced Light Microscopy Facility, using a Leica TCS-SP MP Confocal and Multiphoton Inverted Microscope equipped with an argon laser (488 $\mathrm{nm}$ blue excitation, JDS Uniphase). The images were acquired using a $\times 63$ oil-immersion objective lens and LCS Lite Software (Leica Confocal Software, Leica Microsystems).

Cell-surface biotinylation. Cells were treated with a nonpermeable SH-reactive, maleimide- $\mathrm{PEG}_{2}$-biotin (12) (0.5 mM final concentration) according to the manufacturer's instructions (Pierce). Each ferroportin-GFP construct was analyzed in at least 3 independent experiments.

${ }^{125}$ I-hepcidin internalization assay. The assay was performed as described previously $(12,38)$. Cells were treated with ${ }^{125}$ I-hepcidin for 1 hour at $37^{\circ} \mathrm{C}$. The radioactivity of untransfected cells was subtracted as background for each point. The counts were then normalized to the ferroportin-GFP expression of each sample as determined by quantitation of Western blots using Image Lab Software (Bio-Rad).

Immunoprecipitation and Western blotting. Cell lysis, immunoprecipitation, and Western blotting were performed as previously described (12). Polyclonal ab290 anti-GFP antibody (Abcam) was used for immunoprecipitation, biotinylated proteins were detected with streptavidin-HRPO (Pierce), and a monoclonal anti-GFP antibody (clones 7.1 and 13.1, Roche) was used to determine the amount of ferroportin-GFP that was immunoprecipitated.

Surface plasmon resonance. Surface plasmon resonance was performed on a BIAcore 3000 System (BiaCore AB) as previously described (39). Peptides were immobilized on a CM5 sensor chip by the amine-coupling protocol at a range of response units (1,100-6,000 response units). Analyte solutions were prepared at concentrations of $1-10 \mu \mathrm{g} / \mathrm{ml}$ in HBS-EP buffer (10 mM HEPES, $\mathrm{pH} 7.4,150 \mathrm{mM} \mathrm{NaCl}, 3 \mathrm{mM} \mathrm{EDTA}$, and $0.005 \%$ surfactant P20). Analytes were introduced into the flow cells at $50 \mu \mathrm{l} / \mathrm{min}$ for 3 minutes at $37^{\circ} \mathrm{C}$. Resonance signals were corrected for nonspecific binding by subtracting the signal of the control flow cell and analyzed using BIAevaluation 4.1 software (Biacore).

Peptide synthesis and purification. All peptides were synthesized using the standard solid-phase fmoc chemistry (16) and were purified using reversephase HPLC. Minihepcidins were synthesized as carboxyamides $\left(-\mathrm{CONH}_{2}\right)$, which created a charge-neutral end more similar to a peptide bond than the negatively charged-COOH. The list of minihepcidins with their sequences is shown in Supplemental Table 2, and the structures of unnatural amino acids are displayed in Supplemental Table 3.

Circular dichroism spectroscopy. Circular dichroism spectra (185-260 nm) of the hepcidin peptides were recorded on a JASCO 720 Spectropolarimeter (Easton) as previously described (16). Estimates of the secondary structure contributions were made using the SELCON algorithm (40) and spectral basis set SP43 in the Olis Global Works software suite (Olis).

Modeling of hepcidin-ferroportin interaction. The structure for hepcidin (NP_ 066998, http://www.ncbi.nlm.nih.gov/protein/NP_066998) was modeled by the coordinate set for the lowest energy conformer from the Protein Data Bank (PDB 2KEF) (18). The structure for ferroportin (NP_055400.1, http://www.ncbi.nlm.nih.gov/protein/NP_055400.1) was approximated using the coordinate set provided by Nathan Subramaniam (19). The ferroportin region spanning residues 306-362 and the hepcidin structure were individually minimized in the HyperChem environment to obtain the lowest energy conformer.
Hepcidin was first docked into the ferroportin segment using the initialstage docking program ZDOCK (http://zdock.bu.edu/). The lowest energy conformer set was then used as starting ensemble for a more refined docking using RosettaDock (http://rosettadock.graylab.jhu.edu/), which generated a low-energy structure used as a final hypothetical model for the ferroportin-hepcidin complex.

In vitro bioassay for measuring the activity of hepcidin derivatives. ECR293Fpn (38), a cell line stably transfected with the mouse ferroportin-GFP construct under the control of the ponasterone-inducible promoter, was used for flow cytometry measurements of GFP fluorescence as previously described (16). For each peptide, the treatment with the full range of peptide concentrations was repeated independently 3 to 7 times.

In vivo bioassay for measuring activity of hepcidin derivatives. Animal studies were approved by the Animal Research Committee at UCLA. Six-week-old C57BL/ 6 mice were obtained from The Jackson Laboratory. Mice were placed on an iron-deficient diet ( 4 ppm iron, Harlan Teklad) for 2 weeks to suppress endogenous hepcidin and to decrease its mouse-to-mouse variability $(16,41)$. Mice were subjected to the following treatments: (a) intraperitoneal injection with 0,20 , or 200 nmoles peptide solubilized in $100 \mu \mathrm{l} \mathrm{SL220}$ solubilization agent (NOF) and (b) gavage with 0 or 200 nmoles peptide in $250 \mu \mathrm{l} 1 \times$ solvent (Cremophor EL [Sigma-Aldrich]/ethanol/PBS = 12.5:12.5:75) (42). Mice were sacrificed 4 hours later, and serum iron was determined using a colorimetric assay (Diagnostic Chemicals) (16). To normalize for the variations in absolute serum iron levels among experiments, the results were expressed as the percentage of decrease in serum iron as compared with the serum iron levels in PBS-treated mice. Each peptide concentration was tested in 5 to 7 animals.

For the study of chronic minihepcidin effects, we used hepcidin-1 knockout mice, originally provided to our laboratory by Sophie Vaulont (43). Hamp 1 $1^{-/-}$mice (5-6 weeks of age, 6 males, and 7 females) were placed on low-iron diet for 2 weeks ( $4 \mathrm{ppm}$ iron) to slow down the development of iron overload prior to injections with minihepcidins. The mice were then switched to the standard mouse chow (336 ppm iron) and given $100 \mu \mathrm{l}$ intraperitoneal injections daily of either 100 nmoles palmitoyl-ri-hep9 solubilized in SL220 or the solvent only. After 2 weeks, the mice were euthanized, and their nonheme liver iron concentration was measured. Nonheme iron concentration was determined as described (44), using acid-based protein precipitation followed by a colorimetric assay for iron quantitation (Diagnostic Chemicals).

Quantitative real-time PCR. Liver RNA was extracted using TRIzOL reagent (Invitrogen). Gene expression of Hamp1 and the reference gene $\beta$-actin $(A c t b)$ was assessed by qRT-PCR using the iScript cDNA Synthesis Kit and iQ SYBR Green Supermix (Bio-Rad) (45). HAMP1 primer sequences are as follows: $5^{\prime}$-TTGCGATACCAATGCAGAAGA-3' and 5'-GATGTGGCTCTAGGCTATGTT- $3^{\prime}$. $\beta$-Actin primer sequences are as follows: $5^{\prime}$-ACCCACACTGTGCCCATCTA-3' and 5'-CACGCTCGGTCAGGATCTTC-3'.

Statistics. SigmaPlot version 11.0 (Systat Software) was used for statistical analyses. A $P$ value of less of 0.05 was considered significant.

\section{Acknowledgments}

We would like to acknowledge the Office of Information Technology at the University of California, Irvine, and Broadcom Corp. for providing the Broadcom Distributed/Unified Cluster used for the molecular dynamics simulation and docking of the protein complex. The UCLA Jonsson Comprehensive Cancer Center and Center for AIDS Research Flow Cytometry Core Facility was instrumental for flow cytometry measurements. Matthew Schibler at the UCLA CNSI Advanced Light Microscopy Facility provided help with confocal microscopy. We thank Grace Jung for assistance with Biacore analysis and Nathan Subramaniam for providing the PDB file of 
his ferroportin model. The work was funded by NIH grants R01 DK 082717 (to E. Nemeth) and R01 DK 065029 (to T. Ganz).

Received for publication February 21, 2011, and accepted in revised form September 21, 2011.

1. Ganz T, Nemeth E. Hepcidin and disorders of iron metabolism. Annu Rev Med. 2011;62:347-360.

2. Ganz T, Olbina G, Girelli D, Nemeth E, Westerman M. Immunoassay for human serum hepcidin. Blood. 2008;112(10):4292-4297.

3. Piperno A, et al. Blunted hepcidin response to oral iron challenge in HFE-related hemochromatosis. Blood. 2007;110(12):4096-4100.

4. Origa $\mathrm{R}$, et al. Liver iron concentrations and urinary hepcidin in beta-thalassemia. Haematologica. 2007; 92(5):583-588.

5. Kearney SL, et al. Urinary hepcidin in congenital chronic anemias. Pediatr Blood Cancer. 2007; 48(1):57-63.

6. Fujita N, et al. Patients with chronic hepatitis C achieving a sustained virological response to peginterferon and ribavirin therapy recover from impaired hepcidin secretion. J Hepatol. 2008;49(5):702-710.

7. Girelli D, et al. Reduced serum hepcidin levels in patients with chronic hepatitis C. J Hepatol. 2009; 51(5):845-852.

8. Barton JC. Chelation therapy for iron overload. Curr Gastroenterol Rep. 2007;9(1):74-82.

9. Porter JB. Optimizing iron chelation strategies in beta-thalassaemia major. Blood Rev. 2009; 23(suppl 1):S3-S7.

10. Bartnikas TB, Fleming MD. A tincture of hepcidin cures all: the potential for hepcidin therapeutics. J Clin Invest. 2010;120(12):4187-4190.

11. Rivera S, Liu L, Nemeth E, Gabayan V, Sorensen OE, Ganz T. Hepcidin excess induces the sequestration of iron and exacerbates tumor-associated anemia. Blood. 2005;105(4):1797-1802.

12. Fernandes A, et al. The molecular basis of hepcidin-resistant hereditary hemochromatosis. Blood. 2009;114(2):437-443.

13. Liu XB, Yang F, Haile DJ. Functional consequences of ferroportin 1 mutations. Blood Cells Mol Dis. 2005;35(1):33-46.

14. Rice AE, Mendez MJ, Hokanson CA, Rees DC, Bjorkman PJ. Investigation of the biophysical and cell biological properties of ferroportin, a multipass integral membrane protein iron exporter. JMol Biol. 2009;386(3):717-732.

15. De Domenico I, et al. The hepcidin-binding site on ferroportin is evolutionarily conserved. Cell Metab. 2008;8(2):146-156

16. Nemeth E, Preza GC, Jung CL, Kaplan J, Waring AJ,
Address correspondence to: Elizabeta Nemeth, CHS 37-055, Department of Medicine, David Geffen School of Medicine at UCLA, 10833 Le Conte Ave., Los Angeles, California 90095-1690, USA. Phone: 310.825.7499; Fax: 310.206.8766; E-mail: enemeth@ mednet.ucla.edu.
Ganz T. The N-terminus of hepcidin is essential for its interaction with ferroportin: structure-function study. Blood. 2006;107(1):328-333

17. Clark RJ, Tan CC, Preza GC, Nemeth E, Ganz T, Craik DJ. Understanding the structure/activity relationships of the iron regulatory peptide hepcidin. Chem Biol. 2011 2011;18(3):336-343.

18. Jordan JB, et al. Hepcidin revisited, disulfide connectivity, dynamics, and structure. J Biol Chem. 2009; 284(36):24155-24167.

19. Wallace DF, Harris JM, Subramaniam VN. Functional analysis and theoretical modeling of ferroportin reveals clustering of mutations according to phenotype. Am J Physiol Cell Physiol. 2010;298(1):C75-C84.

20. Chen R, Li L, Weng Z. ZDOCK: an initial-stage protein-docking algorithm. Proteins. 2003;52(1):80-87.

21. Lyskov S, Gray JJ. The RosettaDock server for local proteinprotein docking. Nucleic Acids Res. 2008; 36(Web Server issue):W233-W238.

22. Sham RL, Phatak PD, Nemeth E, Ganz T. Hereditary hemochromatosis due to resistance to hepcidin: high hepcidin concentrations in a family with C326S ferroportin mutation. Blood. 2009; 114(2):493-494.

23. Resh MD. Palmitoylation of ligands, receptors, and intracellular signaling molecules. Sci STKE. 2006;2006(359):re14.

24. Roetto A, et al. Mutant antimicrobial peptide hepcidin is associated with severe juvenile hemochromatosis. Nat Genet. 2003;33(1):21-22.

25. Nemeth E, Roetto A, Garozzo G, Ganz T, Camaschella C. Hepcidin is decreased in TFR2 hemochromatosis. Blood. 2005;105(4):1803-1806.

26. Papanikolaou G, et al. Mutations in HFE2 cause iron overload in chromosome 1q-linked juvenile hemochromatosis. Nat Genet. 2004;36(1):77-82.

27. U.S. Preventive Services Task Force. Screening for Hemochromatosis: Recommendation Statement. Ann Intern Med. 2006;145(3):204-208.

28. Cunningham MJ. Update on thalassemia: clinical care and complications. Pediatr Clin North Am. 2008;55(2):447-460.

29. Taher A, Isma'eel H, Cappellini MD. Thalassemia intermedia: Revisited. Blood Cells Mol Dis. 2007; 37(1):12-20.

30. [No authors listed]. International BioIron society meeting. Am J Hematol. 2009;84(8):E236-E375.

31. Fujita $\mathrm{N}$, et al. Hepcidin expression in the liver: rel- atively low level in patients with chronic hepatitis C. Mol Med. 2007;13(1-2):97-104.

32. Alla V, Bonkovsky HL. Iron in nonhemochromatotic liver disorders. Semin Liver Dis. 2005;25(4):461-472.

33. Nicolas G, et al. Constitutive hepcidin expression prevents iron overload in a mouse model of hemochromatosis. Nat Genet. 2003;34(1):97-101.

34. Viatte L, et al. Chronic hepcidin induction causes hyposideremia and alters the pattern of cellular iron accumulation in hemochromatotic mice. Blood. 2006;107(7):2952-2958

35. Gualdi R, Casalgrandi G, Montosi G, Ventura E, Pietrangelo A. Excess iron into hepatocytes is required for activation of collagen type I gene during experimental siderosis. Gastroenterology. 1994; 107(4):1118-1124.

36. Pietrangelo A. The ferroportin disease. Blood Cells Mol Dis. 2004;32(1):131-138.

37. Gardenghi S, et al. Hepcidin as a therapeutic tool to limit iron overload and improve anemia in beta-thalassemic mice. J Clin Invest. 2010;120(12):4466-4477.

38. Nemeth E, et al. Hepcidin regulates cellular iron efflux by binding to ferroportin and inducing its internalization. Science. 2004;306(5704):2090-2093.

39. Wang W, et al. Retrocyclins kill bacilli and germinating spores of Bacillus anthracis and inactivate anthrax lethal toxin. J Biol Chem. 2006;281(43):32755-32764.

40. Sreerama N, Venyaminov SY, Woody RW. Estimation of the number of alpha-helical and beta-strand segments in proteins using circular dichroism spectroscopy. Protein Sci. 1999;8(2):370-380.

41. Nemeth E, et al. IL- 6 mediates hypoferremia of inflammation by inducing the synthesis of the iron regulatory hormone hepcidin. J Clin Invest. 2004; 113(9):1271-1276.

42. Kim S, et al. Sorafenib inhibits the angiogenesis and growth of orthotopic anaplastic thyroid carcinoma xenografts in nude mice. Mol Cancer Ther. 2007; 6(6):1785-1792

43. Lesbordes-Brion JC, et al. Targeted disruption of the hepcidin 1 gene results in severe hemochromatosis. Blood. 2006;108(4):1402-1405.

44. Pak M, Lopez MA, Gabayan V, Ganz T, Rivera S. Suppression of hepcidin during anemia requires erythropoietic activity. Blood. 2006;108(12):3730-3735.

45. Ramos E, et al. Evidence for distinct pathways of hepcidin regulation by acute and chronic iron loading in mice. Hepatology. 2011;53(4):1333-1341. 